\title{
Alimentación y riesgos para la salud: visiones sobre la alimentación saludable y prácticas alimentarias de mujeres y varones de clase media en el Área Metropolitana de Buenos Aires
}

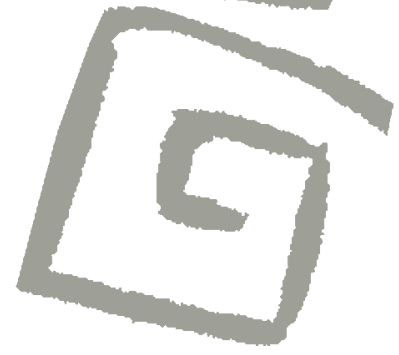

\author{
Food and health risks: views on healthy food and food \\ consumption practices among middle-class women and \\ men in the Metropolitan Area of Buenos Aires
}

\section{Betina Freidin ${ }^{1}$}

${ }^{1}$ Doctora en Sociología. Investigadora independiente, Consejo Nacional de Investigaciones Científicas y Técnicas (CONICET), Instituto de Investigaciones Gino Germani, Facultad de Ciencias Sociales, Universidad de Buenos Aires, Argentina. $\triangle$ (iD)
RESUMEN Analizamos las nociones sobre la alimentación saludable y la percepción de riesgos asociados a la comida industrializada de mujeres y varones jóvenes y de mediana edad de clase media que residen en el Área Metropolitana de Buenos Aires. Los datos provienen de ocho grupos focalizados realizados en el año 2013. Nuestro estudio muestra que los participantes de los grupos incorporan el conocimiento nutricional-científico en sus concepciones de alimentación saludable. Sin embargo, unos pocos plantean los riesgos para la salud derivados del consumo de alimentos procesados, más allá de la creciente divulgación pública sobre las grasas trans y el contenido de sal. Si bien se valoran positivamente los alimentos orgánicos, se objetan su costo y la localización del circuito comercial. Además de la preocupación por la salud, en las prácticas alimentarias intervienen otras prioridades como los costos, la comodidad, la estética, el placer y la sociabilidad.

PALABRAS CLAVES Alimentos Industrializados; Alimentos Orgánicos; Percepción; Riesgo; Clase Social; Argentina.

\begin{abstract}
In this article we analyze notions about healthy food and the perceptions of risks related to industrialized foodstuffs within a group of young and middle-aged females and males who belong to the middle class and live in the Metropolitan Area of Buenos Aires. Data come from eight focus groups that were carried out in 2013. The study shows that the participants of the focus group have incorporated scientific-nutritional knowledge into their conceptions of healthy food. However, few discuss the risks of industrialized food beyond the growing public attention regarding trans fats and salt content. Although organic foods are positively valued, participants object to their high cost and the location of their commercialization. We show how in their food practices, the participants of the focus groups weigh their concern about health against other priorities such as costs, convenience, aesthetics, pleasure and sociability.
\end{abstract}

KEY WORDS Industrialized Foods; Food, Organic; Perception; Risk; Social Class; Argentina. 


\section{INTRODUCCIÓN: LA COMPLEJIDAD DE LA EXPERIENCIA ALIMENTARIA CONTEMPORÁNEA}

Los expertos en ciencias médicas y en nutrición advierten desde hace décadas sobre la centralidad de la alimentación para la prevención de enfermedades y el mantenimiento de la salud. La mayor relevancia en las tasas de morbilidad y mortalidad de las enfermedades crónicas no transmisibles -especialmente, las cardiovasculares, la diabetes, la obesidad, y algunos tipos de cáncer- ha dirigido la atención hacia los factores de riesgo sobre los cuales se espera que los individuos ejerzan control a través de hábitos de vida saludables ${ }^{(1,2)}$. A las recomendaciones para controlar el sobrepeso, la presión arterial y los niveles de colesterol "malo" y glucemia en sangre se suman las orientadas a disminuir el riesgo de contraer enfermedades infecciosas a través de la ingesta de alimentos. La promoción de buenas prácticas alimentarias integra la agenda de los organismos internacionales y las políticas nacionales de salud pública ${ }^{(3)}$, al tiempo que crece la industria de la comida saludable y se tematiza lo alimentario en los medios de comunicación ${ }^{(4,5,6)}$. Entre otras medidas, se destaca el Plan Nacional Argentina Saludable, implementado por la Subsecretaría de Prevención y Control de Riesgos del Ministerio de Salud de la Nación desde el año 2007.

La preocupación por la alimentación requiere de ciudadanos informados sobre temas de salud, que realicen consultas con los especialistas y controlen lo que consumen para sopesar y limitar riesgos ${ }^{(7)}$. Algunos autores conceptualizan la creciente atención brindada al estilo de vida saludable como una dinámica de healthicization, en la que se fusionan las preocupaciones biomédicas con los comportamientos individuales, en un escenario sociocultural en el que gozar de buena salud a través del esfuerzo individual se ha convertido en una virtud ${ }^{(8)}$, especialmente en los sectores sociales medios y altos ${ }^{(1,9,10)}$.

Desde las teorizaciones sobre la sociedad del riesgo o la modernización reflexiva,
Giddens $^{(11)}$ y Beck ${ }^{(12)}$ postulan que las decisiones sobre los regímenes alimentarios operan en un contexto en el que se han diversificado los discursos sobre la alimentación apropiada, y en el que existe una mayor divulgación pública sobre la temática a través de la prensa y las nuevas tecnologías de información y comunicación. Según Lupton ${ }^{(13)}$ :

Es raro ver un día en el que los medios periodísticos no incluyan un informe sobre alguna sustancia en la comida que no se relacione con alguna enfermedad, o que sea protectora para la salud. [Traducción de la autora del original: Hardly a day goes by without a report in the news media eithe on the linking of a food substance with illness or disease, or a claim that a foodstuff serves to protect against ill health].

Asimismo, hay más conocimiento público sobre los riesgos para la salud y el medio ambiente derivados de la producción industrializada de los alimentos. Giddens(11) destaca los cambios en los consensos de los expertos, y cómo en las sociedades industrializadas se incrementan los riesgos para la salud, los cuales tienen un impacto global ${ }^{(11)}$ :

Antes algunos sectores de la profesión médica promovían el tabaco por su efecto relajante, así como la carne roja, la manteca y la crema para tener cuerpos saludables [...]. Un día es el mercurio en los peces, otro es la manteca lo que hay que dejar de comer [...]. Hoy el riesgo está en el fosfato del detergente, mañana en los insecticidas. La amenaza de la muerte, la enfermedad y el cáncer ronda en todo lo que comemos o tocamos. [Traducción de la autora del original: We must recall that smoking was once advocated by some sectors of the medical profession as a relaxant; while red meat, butter and cream were said to build health bodies [...] one day we hear about the mercury and run to throw out cans of tuna fish from our shelves; the next day the food to shun may be butter [...] Today the danger lucks in the phosphates in our favorite detergent; tomorrow the finger points to insecticides. The threats of death, insanity and cancer lurk in all we eat or touch]. 
Fischler ${ }^{(14)}$, por su parte, enfatiza la incertidumbre que generan las formas dominantes de producción y distribución de los alimentos en los centros urbanos: "carecemos incluso de la más mínima idea sobre su origen real, sobre los procedimientos y las técnicas usadas para su producción, expedición y tratamiento". En Argentina, Aguirre ${ }^{(15)}$ retoma las reflexiones de este autor en referencia a las ansiedades e incertezas de los comensales del Área Metropolitana de Buenos Aires (AMBA):

No sabemos qué comemos: si la manzana tiene genes de manzana o si le agregaron otros genes y de qué, si los agroquímicos con que se produjo el alimento no son peligrosos, si los aditivos y conservantes de su procesamiento no son cancerígenos, si está Ileno de sal o azúcar invisibles que no debían estar, si las grasas con que lo procesaron son hidrogenadas, si su transporte fue seguro.

En este contexto sociocultural se observa la expansión global de minorías alimentarias ${ }^{(16)}$ y movimientos sociales que ponen en cuestión la producción industrializada de alimentos por sus riesgos para la salud, el bienestar animal y el medio ambiente, $y$ sus costos sociales ${ }^{(11,12,17,18,19)}$. Se trata de formas de acción individual y colectiva vinculadas con políticas de la vida frente a los riesgos producidos por el capitalismo industrial en el sistema agroalimentario, los efectos de la globalización y las relaciones de poder que estructuran el sistema alimentario(20,21,22). Desde la sociología de la alimentación y de los estudios sobre la cultura del consumo se destacan las prácticas de consumo reflexivo y la preocupación por la calidad de los alimentos ${ }^{(2,17,20)}$, al tiempo que se enfatizan los condicionantes estructurales de las decisiones de consumo ${ }^{(18,23)}$.

Paradójicamente, junto con la mayor divulgación pública de los riesgos que genera la producción masiva de alimentos, la industria alimentaria promueve renovadas versiones de alimentos saludables ${ }^{(18)}$. A los alimentos bajos en calorías y sin grasas "malas" de origen animal (saturadas) y vegetal transformadas (trans), se suman los productos funcionales publicitados por sus propiedades para ayudar a reducir factores de riesgo y malestares crónicos. Por ejemplo, los adicionados con Omega 3 para disminuir el colesterol "malo" y los yogures con probióticos para el "bienestar intestinal". Se trata de un nicho del mercado en crecimiento en el que se desdibujan los límites construidos culturalmente entre los alimentos y los medicamentos $^{(4,15,24)}$.

En un clima generalizado de riesgos e incertezas producto de la deslocalización y mercantilización de la experiencia alimentaria, y de la diversidad de regímenes alimentarios promovidos como saludables, algunos autores plantean que la adopción de un estilo de vida, en tanto prácticas rutinizadas pero abiertas al cambio, permite estabilizar las decisiones sobre qué comer ${ }^{(11)}$. El dilema de qué recomendaciones seguir se resuelve cotidianamente a través de "una mezcla de rutina y compromiso a cierta forma de estilo de vida más la confianza que se deposita en determinados sistemas abstractos. Aunque este 'paquete de compromiso' bajo presión puede comenzar a desintegrarse ${ }^{\prime \prime(11)}$. De manera similar, Fischler ${ }^{(5,14)}$ sostiene que la adopción de un régimen alimentario alternativo (como el vegetarianismo y el consumo orgánico) es una forma de ejercer control sobre la salud frente a la preocupación por los peligros de la alimentación moderna industrializada. Son las clases sociales altas y medias las que tienen mayor margen de elección en las decisiones de consumo alimentario, por su mayor acceso a la información sobre regímenes alimentarios promovidos como saludables y a recursos para implementarlos (dinero, tiempo, accesibilidad a los lugares de abastecimiento, etc. $)^{(1,25,26)}$.

En este artículo analizamos las visiones y las prácticas alimentarias de un grupo de mujeres y varones jóvenes y de mediana edad de clase media, residentes en el AMBA, que entrevistamos grupalmente en el marco del Proyecto UBACyT 20020110200178, Programación 2012-2014. Como los consejos de los especialistas sobre la alimentación en 
relación con la salud se han tornado complejos y difíciles de negociar para los $\operatorname{legos}^{(11,27)}$, y existe una multiplicidad de normas socioculturales sobre el buen comer ${ }^{(15)}$, indagamos sus nociones sobre la alimentación saludable y sus percepciones sobre los peligros asociados a la comida industrializada. Asimismo, damos cuenta de cómo los participantes sopesan la preocupación por la salud frente a otros valores y prioridades como la conveniencia, los costos y la estética, el placer y la sociabilidad. Como los antropólogos y sociólogos lo remarcan, los alimentos tienen un valor simbólico y refuerzan la afiliación social, y en ocasiones las prácticas alimentarias se contraponen a las recomendaciones de los expertos para el cuidado de la salud ${ }^{(15,16,28)}$.

\section{MÉTODOS}

Realizamos ocho grupos focalizados con varones y mujeres de clase media que tenían entre 23 y 50 años (38 participantes en total, con una edad promedio de 32 años). Los grupos focalizados son discusiones planificadas cuyo propósito es indagar las percepciones de los participantes en un área definida de interés en un ambiente permisivo y no amenazante ${ }^{(29)}$. Facilitada por las intervenciones del moderador, el intercambio generado por las intervenciones individuales favorece que los participantes cotejen sus percepciones y puntos de vista. Utilizamos una guía de pautas semiestructurada que permitió focalizar las conversaciones sobre determinados ejes temáticos para el análisis comparativo posterior entre los grupos, así como la emergencia de temas no anticipados ${ }^{(30)}$. De manera complementaria a la guía de pautas, utilizamos materiales audiovisuales como disparadores para la discusión. Incluimos un spot de una campaña del Ministerio de Salud de la Nación sobre alimentación saludable en el trabajo, una publicidad de una galletita con fitoesteroles y un minuto de un capítulo de la serie Los Simpsons sobre alimentos orgánicos.
El trabajo de campo se realizó durante el año 2013. La indagación sobre alimentación y salud fue una de las dimensiones del estudio cualitativo sobre hábitos de vida saludables en sectores de clase media del AMBA. Realizamos cuatro grupos con mujeres y cuatro con varones, buscando homogeneidad en las edades de cada grupo, y distinguiendo grupos de participantes con y sin hijos. Para seleccionar a los participantes consideramos su educación formal (estudios secundarios completos y más) y que desarroIlaran ocupaciones típicas de clase media ${ }^{(31)}$. Como habitualmente se procede en la investigación académica con grupos focalizados con personas de clase media( ${ }^{(4,32,33)}$, los contactamos a través de las redes personales de los miembros del equipo.

Las conversaciones fueron grabadas digitalmente y transcriptas verbatim, tras obtener la autorización de los participantes para hacerlo, mediante la firma de la carta de consentimiento informado, entregada al inicio de cada encuentro. Los nombres incluidos en los extractos de las conversaciones son seudónimos para preservar el anonimato y la confidencialidad. Realizamos un análisis temático cualitativo de los datos, utilizando el programa ATLAS.ti para la codificación y escritura de memos analíticos ${ }^{(34)}$.

\section{RESULTADOS}

\section{La alimentación saludable}

Al preguntarles a los participantes de los grupos focalizados qué era para ellos comer sano o tener una alimentación saludable, ellos refirieron que la dieta sea balanceada y moderada en cantidades, variada en carnes, rica en vegetales y frutas, con pocas grasas "malas" de origen animal y vegetal, y restringida en el consumo de azúcar y sal. La distinción que realizan de los alimentos más y menos saludables también toma en consideración la contraposición entre la comida "chatarra" y la casera. Se ejemplificó la primera categoría con la empresa multinacional McDonald's, ícono de la comida 
rápida y poco nutritiva, con sus equivalentes hogareños de pronta preparación, como salchichas y hamburguesas. Como comida chatarra, algunos participantes identificaron también las golosinas y galletitas, cuando se recurre a ellas para sustituir alguna comida. Similares consideraciones se aplican cuando se abusa del consumo de comidas preparadas fuera del hogar, típicamente a través del delivery. Carlos, en el Grupo 6, comentó que para mantener la salud es importante "alimentarse bien, tratar de no comer mucho afuera, tratar de evitar... tratar de evitar la comida chatarra, rápida y todo eso" y, más adelante en la conversación, agregó que en su casa casi nunca recurre al delivery y que evita la comida "de porquería", "a lo sumo, de vez en cuando, voy a una parrilla y bueno, una vez por mes vamos a comer alguna parrillada a algún lado. Pero, bueno, si no, cocino todos los días". La comida casera se considera saludable porque permite un mayor control en el proceso de selección de los alimentos en cuanto a su calidad y frescura, así como en el seguimiento de pautas de higiene en su elaboración y conservación. En el Grupo 4, Cecilia se refirió a la importancia de esta dimensión de la vigilancia alimentaria:

Cecilia: Yo tengo una historia con los lugares de elaboración. [...] Me mudé al lado de la casa de empanadas, que antes le compraba, y desde que me mudé no les compre nunca más. ¿Entendés? Claro, ves las empanadas, ves el mismo pibe que te la trajo y que tocó todo y que agarró la plata, entra y le hace el repulgue, y te digo no, no compremos empanadas, no compren, las hacemos en casa. (Grupo 4)

Lo "casero" constituye un ámbito de resguardo frente a los peligros para la salud que puede representar el fenómeno creciente de la externalización del consumo alimentario en el $\mathrm{AMBA}^{(6)}$, es decir, el consumo de comidas en restaurantes y bares, así como de comidas elaboradas fuera del hogar entregadas a través del delivery, una práctica muy difundida en los sectores medios. Con límites difusos, el dominio de lo "casero" opera como una fuente de confianza para ejercer control sobre lo que comen ${ }^{(13,35)}$. Si bien se lo asocia literalmente con la comida preparada en el hogar, también se extiende a la elaborada por otros, pero de manera más personalizada y artesanal.

A partir de la demarcación de la alimentación saludable que realizan los participantes, observamos que el discurso alimentario dominante -definido en las guías nutricionales elaboradas por los expertos y difundidas internacionalmente- integra en gran parte su repertorio cognitivo para distinguir los alimentos desde el punto de vista de su contenido nutricional y su aporte para la prevención de enfermedades. Las guías nutricionales tienen como propósito influir sobre los hábitos alimentarios de los ciudadanos para mejorar la salud de la población. En las últimas décadas, los expertos elaboraron la pirámide nutricional y establecieron una jerarquización de los grupos de alimentos y sus cantidades apropiadas que desplazó la centralidad de la carne en la alimentación humana ${ }^{(4)}$. Recientemente, el plato redondo reemplazó a la representación piramidal, dividiendo a los distintos grupos de alimentos en cuatro compartimentos de distintos tamaños (vegetales, granos, proteínas, y frutas) $)^{(18)}$. Argentina lo adoptó en la elaboración de las Nuevas Guías Alimentarias para la Población Argentina, siguiendo los lineamientos del Instituto de Nutrición de Centro América y Panamá y la Organización Panamericana de la Salud (INCAP/OPS). Así es que, por ejemplo, aún cuando algunos no han adoptado la recomendación de disminuir el consumo de sal, reconocen su importancia como práctica de prevención de enfermedades cardiovasculares en el largo plazo. En el Grupo 7, Cynthia comentó que, si bien ella utiliza sal en exceso, tiene presentes los posibles riesgos que ello implica para su salud en el futuro, y no quiere transmitir este hábito a su pequeño hijo:

Cynthia: Yo me siento muy identificada con la sal. (Risas)

Moderadora: ¿Porque usás o porque no usás? 
Cynthia: Porque la uso, y con la excusa de que tengo presión baja le doy a troche y moche. (Risas)

Alicia: Mejor, qué suerte.

Cynthia: No, y bueno, es algo que se usa indiscriminadamente, eso sí.

Moderadora: ¿Pero vos tendrías que consumir menos sal teniendo baja presión? Cynthia: Eh... más que nada por un hábito familiar, no por... porque el nene tiene cuatro años y ya me pide, entonces tengo que hacer de cuenta que le pongo cuando en realidad la comida ya tiene sal al ser cocinada. Entonces es un hábito que no quiero que aprenda.

Alicia: Claro, que agarre el salero porque sí y ya le ponga.

Moderadora: No, yo lo que preguntaba es si a vos te hace mal comer con sal... Cynthia: No. No, no, no. No, por ahora no. Vamos a ver, cuando sea más grande y tenga las arterias un poquito más tapadas, qué pasa. (Grupo 7)

Al igual que en el caso del consumo excesivo de sal, la recomendación de eliminar las grasas trans para prevenir enfermedades cardiovasculares ha recibido importante divulgación pública y, recientemente, las autoridades sanitarias prohibieron su utilización en la industria alimentaria. Las políticas nacionales para la eliminación de grasas transformadas en la industria incluyen la iniciativa "Argentina 2014 libre de grasas trans" y la modificación del Código Alimentario Argentino. Respecto del consumo de sodio, a nivel nacional se destacan la iniciativa "Menos Sal más Vida" para la reducción de la sal en panificados y alimentos procesados, y la Ley 26905 que regula el consumo de sodio.

Los participantes de los grupos contaban con información sobre los peligros que representan las grasas trans en la dieta, aunque pudieran confundir los términos técnicos utilizados para clasificar las sustancias perjudiciales para la salud:

Gabriel: Yo si tengo dos cosas que me resultan ricas, una que no tenga grasas transgénicas, la elijo por sobre la otra.

\author{
Moderadora: Trans. \\ Gabriel: Trans. ¿Eso es transgénica? \\ Moderadora: No. \\ Gabriel: ¿Qué es trans? \\ Moderadora: Trans es... ¿cómo son? \\ Milo: Las malas, saturadas \\ Gabriel: Saturadas. (Grupo 3)
}

Del diálogo anterior se desprende que comprender las distinciones técnicas y leer las etiquetas de información nutricional de los envases requiere un aprendizaje o "alfabetización" por parte de los consumidores para poder tomar decisiones de compra informadas, e indica una actitud de vigilancia respecto de la calidad de los productos procesados $^{(35)}$. En el Grupo 4, las mujeres articularon la necesidad de estar informadas tras experiencias negativas de compra de productos de dudoso valor nutricional, aun en las grandes cadenas de supermercados, donde cabría esperar que desarrollen políticas comerciales que prioricen la calidad de los productos que exhiben en las góndolas:

\section{Rosario: El que me indigna a mí es Hamlet, la golosina Hamlet. Yo digo: no puede ser que eso lo vendan ahora en las grandes cadenas de supermercados, que lo pongan ahí con las golosinas, y no tiene nada de chocolate, cero chocolate tiene. \\ Cecilia: Ah, claro. \\ Rosario: Es aceite hidrogenado. (Grupo 4)}

El discurso médico-científico sobre la alimentación saludable es divulgado por los medios masivos de comunicación, transmitido en las consultas clínicas, además de circular de boca en boca en los distintos espacios de interacción cotidiana. En todos los grupos los participantes recordaban la presencia de médicos y especialistas en nutrición en programas televisivos dedicados a la alimentación, o incluso de cocina, y en las columnas de los noticieros. También mencionaron programas radiales, periódicos, revistas y sitios de Internet. En el Grupo 5 se utilizó el término "bombardeo" para referirse a la creciente cobertura periodística sobre la vida saludable que observan en los distintos medios 
audiovisuales. Si bien se objeta la sobreabundancia de mensajes y recomendaciones en los medios de comunicación masivos, también se valora su función social educadora en materia alimentaria. Así, las mujeres del Grupo 4 mencionaron como beneficiosos los programas de salud que ofrecen información sobre la dieta para prevenir enfermedades cardiovasculares, al tiempo que demandaban mayor divulgación sobre pautas de higiene para evitar el contagio de enfermedades infecciosas durante la preparación y conservación de los alimentos. También se espera que el Estado intervenga más activamente a través de campañas públicas de promoción de la salud que ayuden a los consumidores a identificar publicidades engañosas de productos que contienen sustancias perjudiciales para la salud. Por ejemplo, Leo sostuvo: "yo no veo anuncios que digan, no tome más gaseosa porque te pudre los dientes, empiecen a tomar más agua". (Grupo 3)

Al demarcar el dominio de la alimentación saludable en algunos grupos también surgió la referencia a nuevas tendencias alimentarias. Fue en el Grupo 2, de mujeres más jóvenes, donde mayor énfasis se puso en nuevas modalidades de cuidado, o lo que ellas denominaron como una "moda" alrededor de la comida saludable, asociándola con el movimiento de la alimentación natural y la cultura green de cuidado del medio ambiente. Aunque observaron una creciente cobertura en los medios de comunicación masivos, no obstante destacaron que la información sobre estas nuevas modalidades de consumo y estilos de vida circula fundamentalmente en sus redes sociales e Internet. Esto no es sorprendente, ya que los expertos en comunicación muestran la importancia de las nuevas plataformas digitales para el intercambio de información sobre la salud y el bienestar, que desafían los discursos dominantes y pluralizan los saberes públicos ${ }^{(36)}$ :

Moderadora: Y, y de eso que hay... básicamente... ¿De qué medios recuerdan? Mencionaron la radio, la tele...

Verónica: ¿De lo naturista?

Moderadora: Sí.
Verónica: De boca en boca, para mí. Valeria: Sí. [Juana y Micaela también acuerdan]

Valeria: Para mí también, de boca en boca, redes sociales... revistas digitales que hablan de lo verde y eso, que quizás leo por temas de reciclaje. También es cómo llevar una vida sana y mercados viste... que podes tener frutas que no fueron intervenidas con pesticidas, o lo que sea, conservantes, etcétera. Sabe la Tierra en San Fernando, o varios mercados así que son como más eh... naturales, lo que compras y eso es todo lo que lo llamo moda verde que está bueno que pase si la gente se copa, porque es para beneficiarte la salud, digamos. (Grupo 2).

Solo unos pocos cuestionaron las recomendaciones dominantes sobre la alimentación, tratándose especialmente de los participantes con mayor experiencia personal en el seguimiento de regímenes alimentarios alternativos, y de aquellos que contaban con mayor información sobre los debates y disensos de los expertos sobre la alimentación protectora de la salud. En las conversaciones grupales, plantearon la importancia de mantener una distancia crítica frente a algunas recomendaciones sostenidas tanto por el discurso médico-científico como por la industria alimentaria. Por ejemplo, Mariana, en el grupo 2, que se encontraba en un proceso de transición hacia el vegetarianismo, consideró que la mayor información sobre opciones de estilos de vida demanda consumidores reflexivos, al tiempo que abre la posibilidad de empoderamiento frente a las presiones de la industria alimentaria al pluralizarse las opciones de consumo:

\footnotetext{
Mariana: Al haber tanto estímulo y tanto consumo, como que hay que empezar a elegir y... bueno, entre tanto, eh, tanta información, estás obligado a decidir y, capaz antes no tanto. Para mí es eso, que hay más conciencia y la gente lo sabe, entonces ya no te venden cualquier cosa. [...] Y... hace un año que soy vegetariana, por ejemplo, y para mí es traer mucha más conciencia a lo que como, a lo que elijo comer. Después,
} 
mi novio también, en la familia comen todo orgánico, eh... entonces, eh... no toman pastillas, eh... consumen, eh... medicamentos naturales y cada vez veo más gente que consume, eh... cosas naturales. (Grupo 2)

\section{Prioridades en conflicto en las decisiones de consumo}

Las madres con hijos en edad escolar expresaron su preocupación por el contenido nutricional de los alimentos que componen el segmento del mercado destinado a los niños, y que utiliza estrategias de micromarketing para asociar su consumo a la diversión $^{(18,37)}$. Ellas mantienen una actitud de vigilancia frente al "bombardeo" de la oferta, pero también de negociación. Por ejemplo, en el Grupo 4, manifestaron que regulan su compra y optan por la preparación de repostería casera, reservando para los encuentros sociales de sus hijos el consumo de los productos que encuentran inconveniente. Así, buscan preservar la sociabilidad de los niños con sus pares, habida cuenta de que los alimentos tienen un valor simbólico y constituyen un medio para reforzar la afiliación y la pertenencia grupal ${ }^{(16,28)}$ :

Susana: Pasa que estás bombardeada por la oferta, el comercio... Yo pensaba en los chicos, los chicos es un mercado, actualmente, de elite [...] los lácteos, las golosinas, las galletitas, los cereales...

Rosario: Lo que trato de hacer con mi familia y lo que personalmente hago es, bueno, qué sé yo, por ejemplo, miro mucho los ingredientes de las cosas que compro, leo siempre el tema de las grasas trans, el aceite hidrogenado [...]. Así que, qué priorizo, y bueno, principalmente esto que te decía, que la comida sea lo más casera posible, que dentro de todo... o sea, yo hago una torta y le pongo manteca, y bueno, es así. [...] Pero por ahí vienen los amiguitos de los chicos y traen un paquete de algo, y bueno, se comen eso. Es también un equilibrio [...] [para] que tengan una vida social razonable. (Grupo 4)
Siguiendo la línea argumental de DeVault sobre las mujeres como cuidadoras del hogar a través del feeding work, Koch ${ }^{(38)}$ sostiene que en la medida en que la alimentación se conecte con la salud, el bienestar y, últimamente, con la vida, se trata de una responsabilidad que recae en las madres. Esta expectativa respecto del rol de las mujeres madres de niños y pre-adolescentes, como vimos, se negocia cotidianamente en el hogar. Elliot $^{(37)}$ señala que, con el crecimiento del mercado de productos para el consumo infantil, actualmente los niños pueden influenciar en un importante porcentaje las decisiones de compra de alimentos. De este modo, se revierten en gran parte, como Aguirre ${ }^{(39)}$ lo advierte, las tendencias históricas de la decisión alimentaria en el grupo familiar. Nuestro estudio muestra que las madres ponen en marcha estrategias de balance para conciliar demandas de cuidado en conflicto, como lo son la atención al componente nutricional de los alimentos y las preferencias de los hijos por determinados productos promocionados por la industria y que comparten habitualmente con sus pares.

Algunas mujeres ponen en cuestión, asimismo, los productos de rápida preparación que ofrece la industria para el menú hogareño. Una de las participantes remarcó que esta decisión va a contramano de los mensajes publicitarios que promueven la imagen de la madre moderna que utiliza productos procesados para preparar comidas sabrosas con poco esfuerzo. La frase "Nunca voy a ser mamá Luchetti" [imagen publicitaria de una marca de pastas] condensa la decisión de Rosario, en el Grupo 4, de no conformar al modelo materno funcional a los intereses de la industria de los alimentos de rápida preparación, recuperando el valor de las formas tradicionales de cocinar. Vemos, de este modo, cómo los consumidores activamente interpretan y transforman los significados simbólicos codificados en las publicidades para poner de manifiesto sus circunstancias personales y sociales, así como su trabajo identitario y propósitos de estilo de vida ${ }^{(40)}$.

Por el contrario, las participantes del Grupo 7 plantearon la conveniencia de relajar 
la vigilancia alimentaria para conciliar entre lo rico y fácil de preparar, y lo saludable y laborioso en la elaboración del menú familiar. En este marco de negociación de prioridades en conflicto enmarcaron positivamente el recurso del delivery y la comida chatarra. Una de las participantes utilizó la noción del "día permitido" para referirse a la relajación de la disciplina alimentaria como un ritual familiar de disfrute ${ }^{(41)}$. Cocinar "sano" a diario constituye generalmente una tarea femenina invisibilizada, que es tiempo-intensiva y que requiere la planificación del menú y ocuparse de la provisión ${ }^{(39,42)}$. Recurrir a la comida chatarra y al delivery de manera excepcional constituye una licencia que se "permiten" y que, por lo tanto, refuerza el carácter moral del régimen alimentario saludable que las mujeres-madres buscan implementar:

Cynthia: No, y eso de decir... una vez por semana decir bueno, a partir de ahora en esta casa se empieza a comer saludable, y al otro día no tengo ganas de cocinar y pido una pizza. Es difícil de mantener, con el tema de las corridas, de que uno siempre está apurado, cocinar sano, y no terminar agarrando la hamburguesa o las salchichas que tenés en el freezer.

Gabriela: Sí, lleva como mucho más tiempo, mucha más dedicación.

Cynthia: Porque vos cuando vas al supermercado sabés que hay días que no tenés tiempo, entonces ya las comprás de antemano, sabés que en algún momento las vas a usar. Es un trabajo extra cocinar "sano".

Alicia: Yo me tengo un "permitido". Me lo hice tipo en el calendario, los viernes al mediodía en mi casa se comen panchos. Es así, fijo, ese día yo no cocino. [...] Pero todo el resto de la semana "se cocina"... me permito una escapada. Esta [vez] no, bueno, se come comida chatarra, listo. (Grupo 7)

Las mujeres y varones más jóvenes que viven solos y trabajan a tiempo completo, o trabajan y estudian, apuntaron no solo a la falta de tiempo sino también al desincentivo de cocinarse y organizar una rutina alimentaria cuando no se comparte con otros, optando por la conveniencia de la comida externalizada.

\section{Cambios en los consensos de los expertos y los intereses de la industria}

Algunos participantes señalaron los cambios a lo largo del tiempo de los consensos de los expertos sobre la alimentación apropiada como una fuente de incertidumbre sobre qué consejos seguir. El ejemplo discutido es la recomendación de disminuir el consumo de carne roja para prevenir enfermedades cardiovasculares. Como se desprende del siguiente testimonio, el carácter provisional y revisable del conocimiento científico $^{(1)}$, si bien genera más incertidumbre sobre las prácticas alimentarias, también sirve como argumento para relativizar algunas recomendaciones y no seguirlas, e ironizar sobre las potenciales consecuencias al asumir que "de algo hay que morir":

Carlos: Sí, bueno, el médico antes decía que fumar era bueno...

Patricio: Pero te están recomendando variado, no te dicen "no comas carne". Carlos: Porque está de moda.

Andrés: No, no sé.

Carlos: Ponen algo de moda y lo ponen de moda.

Patricio: El cerdo, en algunos casos, está más barato que el corte de carne de vaca... y recomiendan comer carne de cerdo.

Moderadora: Sí, van cambiando, ¿no? Las recomendaciones...

Patricio: Sí, es verdad, bueno, mañana te dicen que comer carne de cerdo es cancerígeno y cagamos, pero bueno... algo tan extremo.

Andrés: De algo hay que morir. (Risas)

(Grupo 6)

Siguiendo a Lupton ${ }^{(13)}$ y Ward et al.(2), una respuesta posible frente a los cambios en el discurso médico-científico sobre la alimentación saludable es ignorar de plano las recomendaciones y confiar en el propio juicio 
sobre la razonabilidad de las decisiones alimentarias. Como plantean los autores ${ }^{(2)}$ :

...la culpa se atribuye a la comunidad científica por el cambio en las ideas sobre la "verdad" respecto de los alimentos, así como también a los medios de comunicación por contribuir al pánico alimentario. [Traducción de la autora del original: the "blame" was laid at the feet of both the scientific community for changing ideas about the "truth",

and the media for scaremongering].

El cuestionamiento y las dudas sobre las recomendaciones nutricionales dominantes también responden a una actitud más generalizada de alerta cuando se sospecha que pueden estar alineadas con los intereses de la industria alimentaria. Es el propio disenso en la comunidad científica lo que lleva a prestar atención a los intereses comerciales respecto de los cuales las guías nutricionales pueden ser funcionales. Susana, en el Grupo 4, aclaró que "sospecha de todo" y comentó cómo sus dudas sobre la alimentación de los niños se incrementaron a partir de la lectura de un artículo médico en una revista infantil:

Susana: Había una nota, justo la leí, que hablaba de la pirámide alimenticia, sobre todo en los niños, y que estaba interesante, que hablaba, claro, decía que el piso, que te pone que son los cereales... y que decía que... él [un médico] que decía estaba erróneo, que en realidad en el trasfondo hay un... lo de siempre en este mundo, el comercio, la demanda, lo que más vende, porque para él, este se posicionaba, que para él eran las verduras, las frutas, y no las harinas, los cereales, que son de colores, de sabores, de esto, de aquello. (Grupo 4).

La experiencia personal con enfoques alternativos que priorizan la "alimentación como medicina" también constituye una fuente de conocimiento para sostener posturas críticas acerca de las recomendaciones dominantes, como el consumo de lácteos en niños y adultos. Rosario, en el Grupo 4, que tiene una extensa trayectoria experimentando con dietas naturistas, macrobióticas y el crudismo comentó:
Rosario: Muchas veces me encuentro con que todas las... con que muchas de las recomendaciones estoy netamente opuesta, o sea en contra. Eh... consumo de lácteos creo que no... muchas de las cosas que recomiendan como sanas... me parece que todo es mejor que la alimentación convencional de la tostada con leche a la mañana. (Grupo 4)

\section{Los productos light y los funcionales}

Aunque en casi todos los grupos tanto las mujeres como los varones consumían productos light por preocupaciones estéticas, especialmente las mujeres más jóvenes, algunos participantes refirieron las dudas que les generaban las etiquetas de información nutricional de estos productos y el conocimiento técnico que se requiere para entenderlas. También criticaron los elevados precios de este nicho del mercado. Pusieron en cuestión la lógica lucrativa de la industria al promoverlos como saludables frente a opciones más económicas y tradicionales (comer de todo pero con moderación), y cómo los especialistas pueden ayudar a desmitificarlos. Beatriz, en el Grupo 7, concluyó que los productos light son "un engaño", siguiendo los consejos de la nutricionista del marido que lo asesora para adelgazar y que "le enseñó a leer las etiquetas" en el supermercado, ya que si no "vos le ves la etiqueta verde y [lo] agarrás". Observamos que el conocimiento experto constituye un recurso que los sujetos movilizan en sus dinámicas de re-skilling para poder actuar como consumidores críticos frente a las fuerzas del mercado ${ }^{(1)}$. También reclamaron la intervención del Estado a través de las agencias reguladoras encargadas de controlar la veracidad de los mensajes publicitarios y la información contenida en los envases de los productos: 
Diego: El problema es la falta de auditoría que hay en cuanto a políticas públicas y demás. No puede ser que uno se dé cuenta después, del Chocoarroz, que fue un boom, por ejemplo, y todos decían que no engordaba nada, y ahora parece que le encontraron que engorda más que un alfajor. (Grupo 5)

Ahora bien, si la confianza en los especialistas facilita el empoderamiento para las prácticas de consumo frente a un mercado alimentario que genera muchas sospechas, lo cierto es que la confianza depositada en ellos dista de ser ciega. Retomando a Giddens ${ }^{(11)}$, la relación entre los legos y los expertos en la modernidad tardía es conflictiva, y en ella coexisten elementos de fe, confianza, duda y escepticismo. Así es que si bien, por un lado, se confía en las ciencias de la nutrición como fuente de información "objetiva" frente a la motivación de lucro de las corporaciones alimentarias, por el otro, se duda de los intereses personales de los expertos. Especialmente, cuando las recomendaciones las dan médicos con gran exposición mediática y que comparten intereses con la industria en la promoción de determinados productos. En estos casos, sus consejos son objeto de sospecha:

Diego: No sé si fue [el Dr.] Cormillot, que capaz que no le dieron el billete... (Risas) Yo lo escuché hablar a él, y lo tiró abajo al Chocoarroz.

Moderadora: ¿Sí?

Diego: Lo hundió. Y el turrón...

Moderadora: ¿En serio?

Diego: Y levantó el turrón, porque

el turrón... y ahora un boom de

turrones por todos lados. (Grupo 5)

En tanto alimentos industrializados potencialmente riesgosos para la salud, los productos light despertaron opiniones mixtas. A la pregunta de la moderadora acerca de si eran saludables, los participantes respondieron que lo eran cuando tenían un bajo o nulo componente de grasas "malas". Sin embargo, también apuntaron a su posible toxicidad, refiriéndose especialmente a los edulcorantes artificiales. Los que los consumían habitualmente expresaron las ambivalencias que les generaba tener que dirimir entre la preocupación por lo sano y por el peso ("lo sano también engorda", Grupo 1), mientras optaban por utilizarlos debido a sus beneficios estéticos.

La información difundida a través de los medios acerca de los efectos cancerígenos de la sacarina les permite a algunos justificar su preferencia por los alimentos que "engordan" (con alto contenido de azúcar) y, a otros, abstenerse de utilizarla, pero seguir consumiendo otros productos light para los cuales aún no hay evidencia científica respecto de su peligrosidad para la salud. Por ejemplo, el edulcorante artificial no nutritivo aspartame o aspartamo fue incorporado al Código Alimentario Argentino con una ingesta diaria admitida de hasta $40 \mathrm{mg} / \mathrm{kg}$ del peso corporal. Debido al pánico generado por la información difundida sobre su peligrosidad en distintos medios sociales e Internet, hace unos años la Administración Nacional de Medicamentos, Alimentos y Tecnología Médica (ANMAT) dedicó un artículo a su uso seguro según la investigación científica disponible y las regulaciones internacionales ${ }^{(43)}$.

El rechazo cognitivo frente a la artificialidad de los productos light y el temor por su potencial toxicidad, además del disgusto por el sabor, también conducen a algunos participantes a desestimar su consumo. Así es que Rosario, en el Grupo 4, no compra productos light porque los considera malos para la salud: "prefiero comerme un kilo de azúcar y no un kilo de aspartame [...]. A mí no me gusta porque creo que es malo".

Las críticas se extendieron a los alimentos funcionales. Como lo plantea Chen $^{(24)}$, los alimentos convencionales y los productos medicinales, en principio, constituyen categorías culturales diferenciables en Occidente, y esta distinción lleva a que los productos funcionales o nutraceuticals introduzcan interrogantes acerca de la "alimentación como medicina". El estatus liminal de los alimentos funcionales efectivamente despierta dudas entre los participantes y genera 
sospechas acerca del interés de la industria en expandir sus ventas creando "nuevos" enfermos y dependencia de productos con un estatus incierto:

Diego: Tenés, por ejemplo, que a veces te crean como una enfermedad. Tenés el caso de La Serenísima, que tiene el Actimel, o alguno que tomás por el tema del colesterol, y no es cuestión de clavarte 10 de esos del colesterol para bajarlo, ¿no? Primero, tenés que ir a un médico y demás. Por eso, yo veo que a veces te crean una enfermedad, ellos te dan la cura y es muy agresivo todo el tema comercial, del mensaje de los medios, ¿no? Con que te quieren imponer esas cosas. [...] La Serenísima, tiene el Actimel, el Activia, por ejemplo, y vos no tenés una persona mayor que no te consuma un Activia. ¡Vas al supermercado y todos tienen problemas! (Grupo 5)

Una vez más, la consulta médica es vista como un recurso de resguardo frente a los posibles riesgos para la salud de las estrategias de marketing de las corporaciones alimentarias. En línea con los trabajos sobre "audiencias activas", esta actitud da cuenta de la atención crítica de los consumidores ante determinados productos y marcas ${ }^{(44,17)}$. Ahora bien, aunque los productos funcionales fueron objeto de sospechas, los participantes valoran su utilidad para algunas situaciones de salud específicas, por ejemplo, para fortalecer el sistema inmunológico y la flora intestinal:

Cecilia: Yo los uso [Actimel] cuando están con... cuando los veo con bajas defensas. Consumen una temporada. Porque en realidad Sandro tuvo alguna vez... este... ¿Cómo se llama?... Bueno, se agarró una ETA [enfermedad transmitida por alimentos] que nadie se la descubría, se agarró una bacteria. Y estuvo con varios episodios, hasta que descubrieron que era eso, que, bueno, fue una consulta telefónica con mi amiga [médica generalista], y un coprocultivo, como correspondía, que nadie se lo mandaba a hacer. Este... me dijo que mientras tanto le diera... que ella es enemiga del Actimel, qué sé yo, pero bueno, pero eso... Pero le benefició, digamos, de alguna manera fue como levantando las... le permitió entablar mejor la lucha con la bacteria [...]. Ahora Gerardo [el marido] que está con... le traje, incluso se lo traje descremado porque está con esos intestinos, pobre, que los viene arrastrando, entonces le digo: bueno, tomate alguno de estos, y están ahí esperando, y los chicos por ahí en las épocas que se dice... Agustín [el hijo] ahora que está con resfriados se toma algunos, pero no es algo que tomemos habitualmente. (Grupo 4)

Incluso algunos consumen ocasionalmente yogures con probióticos porque les gusta su sabor, sin prestar atención alguna a las propiedades protectoras para la salud a partir de las cuales se los promociona. Por ejemplo, Enrique sostuvo: "Yo a veces me agarro uno [Activia] porque es rico el gusto, pero no porque... no me fijo para qué es...". (Grupo 8).

\section{Los alimentos orgánicos, las minorías alimentarias y las preocupaciones ético- ambientales}

La crítica de los participantes de los grupos a la industria alimentaria no solo se basó en las sospechas sobre sus estrategias de marketing, sino que apuntó también a los posibles riesgos para la salud que conllevan los alimentos procesados y la tecnologización de la producción agropecuaria en términos más globales. Se trata de los "bads" o consecuencias negativas de los procesos de industrialización que tienen un impacto global ${ }^{(12)}$, y que generan estados de incerteza y ansiedades alimentarias para los sujetos por la creciente información pública sobre los riesgos para la salud ${ }^{(14,1)}$.

La atención a este conjunto de riesgos fue mayor entre los participantes de los grupos con más información sobre las características de los alimentos orgánicos. Las referencias no fueron sólo a las grasas trans utilizadas en los alimentos industrializados, como vimos 
en los apartados anteriores, sino también a la manipulación genética, agrotóxicos, conservantes, colorantes y hormonas, entre otros procesos tecnológicos que "desnaturalizan" los alimentos y ponen en riesgo la salud humana. A la alteración del gusto y de la estacionalidad de la producción de los alimentos y de su ciclo de vida, se suma el riesgo de toxicidad. Esto lleva a disminuir o evitar su consumo, e incluso a ironizar sobre los posibles, e inciertos, efectos sobre el organismo. Cecilia, en el Grupo 4, hizo referencia a alimentos que se consideran saludables desde el punto de vista nutricional, como el pollo y la soja, pero que en los últimos años se han transformado en productos emblemáticos de la peligrosidad potencial de los alimentos producidos por la agroindustria:

\section{Cecilia: Me pasa con los pollos [de granjas industriales], que me parece que es el alimento que está en peor estado, así en situación general, digo, que no sabemos qué comemos cuando comemos pollo. Yo dejé de comer cosas con soja, yo comía y era base de la alimentación, y el tofu ese que es horrible y qué sé yo, y yo te digo, no te como nada con soja. \\ Moderadora: ¿Por qué? \\ Cecilia: $Y$, no tenés una soja normal, es transgénica. (Grupo 4)}

Cecilia continúa explayándose sobre los vegetales "denaturalizados" que han perdido su sabor respecto de sus equivalentes tradicionales en el pasado ${ }^{(45)}$ y que han sido objeto de procesos de polución química y biológica(46) Menciona los "tomates con sabor a pescado" y las sospechas que le genera la durabilidad artificial de la leche fluida. La participante ironiza sobre los posibles efectos ("iremos mutando") en el cuerpo humano como consecuencia de la intervención tecnológica sobre la naturaleza:

Cecilia: La leche tiene tanto químico que no vence nunca, es eterna también. Entonces lo que estás tomando por ahí difiere radicalmente con lo que es leche, pero bueno, iremos mutando. [...] A mí me parece que igual también hay una cosa con la calidad de los alimentos, más allá de estos alimentos que uno puede seleccionar, sino que la verdura que vas a comer tampoco es una verdura que esté cuidada, ni que esté... [...] lo que te digo es: uno evita, evitás un millón de cosas, y cuando te comiste algo tenía una cantidad de plaguicida que el cuerpo lo tiene que asimilar. (Grupo 4)

Del testimonio anterior se desprende, como lo sintetiza Fischler -citado en Lupton $^{(4)}$ - que para los consumidores urbanos contemporáneos los peligros asociados a la alimentación ya no derivan solo del miedo a la descomposición biológica sino, y fundamentalmente, de los aditivos químicos, rastros de sustancias tóxicas o el procesamiento excesivo.

En contraposición a los productos "desnaturalizados" de la agroindustria, los alimentos orgánicos -que varios de los participantes tuvieron dificultad para definir- son vistos positivamente por su sabor y frescura, así como por no representar riesgos para la salud. Si bien todos reconocen la superioridad de su sabor y calidad nutritiva, se objetan los precios y la dificultad para comprarlos debido a la localización de los lugares de provisión, que contrasta con la mayor accesibilidad de los productos comunes de distribución masiva. Se señala el carácter elitista de este segmento del mercado de alimentos, como ocurre en otros contextos nacionales ${ }^{(23)}$.

De acuerdo con Aguirre ${ }^{(39,26)}$, la producción orgánica se expandió en Argentina en la década de 1990, colocándose mayormente en el mercado internacional. Para el año 2006, los costos de los productos orgánicos en el mercado local superaban en un $20 \%$ o $30 \%$ a sus equivalentes regulares. Estimamos que la brecha en los precios de algunos productos orgánicos sea aun mayor en la actualidad, aunque existen algunas experiencias de venta directa productorconsumidor en ferias alternativas en las que se privilegian los bajos costos, y en las que algunos productores agrupados en cooperativas solo ofrecen productos que responden a los criterios ético-solidarios del comercio 
justo $^{(47)}$. En esta dirección, el Programa ProHuerta, implementado por el Ministerio de Desarrollo Social y el Instituto Nacional de Tecnología Agropecuaria (INTA) desde hace 25 años, busca promover el desarrollo a nivel nacional de huertas agroecológicas para el consumo familiar y comunitario en los sectores sociales más vulnerados a partir de una política de inclusión social y promoción de la salud.

Algunos participantes habían optado por el consumo de vegetales y frutas orgánicas en el pasado, pero lo discontinuaron por cuestiones de costo y de conveniencia. Este tipo de consumo, difícil de sostener en el tiempo, además deja en evidencia los límites de las prácticas de control que pueden implementarse en el nivel individual y familiar frente a los peligros para la salud que representan las formas dominantes de producción de alimentos, y las amenazas del entorno físico urbano en el que transcurre la vida diaria. Estos riesgos requieren de soportes colectivos y de políticas públicas de control alimentario y de cuidado del medio ambiente, así como de responsabilidad corporativa, frente a los procesos crecientes de degradación y polución del entorno ecológico urbano ${ }^{(46,48,49)}$. Atentas a estas condiciones para alcanzar una vida urbana más saludable, Rosario y Cecilia relativizan el alcance de los cambios alimentarios que ellas intentaron implementar en algunos momentos, y apuntan a la contaminación ambiental a la que están expuestas al vivir en un barrio céntrico de clase media y media-baja de la ciudad de Buenos Aires:

Cecilia: Yo viví catorce años al lado de la autopista, o quince, o dieciséis, con lo cual si no te agarra una peste, un... algo cerebral...

Graciela: Sí, sí, también...

Rosario: Porque no podrías respirar cuando... O sea, yo salgo acá en una calle que pasan tres líneas de colectivos, y cuando voy a la mañana, me levanto, y salgo y voy a trabajar... o sea, ¿de qué me sirvió la cosa orgánica...? (Grupo 4)
En otros grupos, algunos participantes desconfiaron de los mecanismos locales de certificación de la producción orgánica, además de cuestionar el costo de los productos, lo que los llevó a desestimar su consumo. También se criticaron las posturas extremas frente a las ansiedades alimentarias. Mara, en el Grupo 2, sostuvo: "Pero además, o sea, lo que yo te digo, el orgánico extremo, ves que todo te hace mal, todo es cancerígeno, todo. Y bueno, está bien, pero frená".

Fue excepcional la referencia a los costos ambientales y al sufrimiento animal de las formas de producción agroalimentaria dominantes. En el Grupo 3, el tema fue introducido por Milo, al relatar su decisión de convertirse al vegetarianismo y optar por el consumo orgánico. Lo describió como un proceso de toma de conciencia sobre los distintos niveles de daño que produce el sistema de producción agroalimentario dominante. Milo, que cursaba un posgrado en ecología y energías renovables, puso el foco en la necesidad de estar informado para tomar conciencia de la dimensión ética y política de las decisiones de consumo alimentario:

Milo: Me hice vegetariano y, como trato de comer orgánico... y... en general vegetales de granja, si es posible, bah, no de supermercado digamos, si es posible. $Y$ semillas y granos y eso.

Moderadora: ¿Y por qué empezaste? Milo: Por un lado, y principalmente, por un desprecio hacia la industrialización... sea de animales o sea de empresas y pesticidas y..., por otro, porque... no, básicamente, por cuestión de cuidado personal, ¿no? Pero mínimo, más que nada por una cuestión de conciencia en cuanto a lo que es la industrialización de la alimentación que le estoy muy en contra. [...] Respecto a la mierda que comemos cada día y respecto al sufrimiento de los animales. [...] creo que muchos de nosotros, si supiéramos las cosas que les hacen a los animales que comemos en un asado, dejarían de comerlos [...]. Mucho más en profundidad con lo que son vegetales y pesticidas o lo que sea, ¿no? Pero... creo que lo principal 
es tener información, como decís, y ahí es que te cambia la cabeza y hacés las cosas que... o podés tomar decisiones. (Grupo 3)

La proclama de Milo a favor del consumo alimentario sustentable y que elimine la crueldad hacia los animales dio lugar a un debate en el grupo. Se remarcó la dificultad de dejar de comer carne en la cultura gastronómica local caracterizada por ser muy carnívora, y en la que la carne roja se asocia con el placer personal y con rituales de comensalidad masculinizados (el asado) ${ }^{(50)}$. Como lo expresó Gabriel, en el grupo 3: "para mí comer también es un placer... fundamental. Entonces, no solo voy a comer como una maquinita de acuerdo a lo que a mi organismo mejor le vendría". Frente a los productos orgánicos, también se priorizaron los menores costos y la accesibilidad de los productos regulares de venta masiva.

$\mathrm{Al}$ evaluar al vegetarianismo y el veganismo como opciones alimentarias, varios participantes señalaron la necesidad de contar con un capital cultural ad hoc -esto es, información para poder sustituir los productos de origen animal sin incurrir en déficits nutricionales- lo cual les demandaría esfuerzo personal y tiempo para la resocialización alimentaria. Incluso, en el Grupo 5 , se bromeó acerca de que sus adeptos constituyen "sectas", o se expresaron prejuicios negativos y rechazo ante las minorías alimentarias:

Alicia: Ni en pedo dejo de comer carne [...] me parece medio repugnante, viste el macrobiótico, que mete todo en la licuadora y todo sale verde o color marrón, dejame. Moderadora: ¿Dónde viste eso?

Alicia: En películas, y alguna loca que conocí.

Cynthia: Yo probé, cuando trabajaba en microcentro probé pasto. Trabajaba en el microcentro, y ponen esos locales top para hacer el boom de lo orgánico, y probé. Moderadora: ¿Qué probaste?

Cynthia: Pasto.

Alicia: ¿Pasto?

Cynthia: Pasto, "grass" se Ilama, o... no me acuerdo, licuado, y lo probé...

Beatriz: ¿Pasto, en serio?

Cynthia: Pasto, pasto, sí. No sé, el lugar se llamaba Punto Fresco, o... bueno, sí.

El jugo era ese jugo verde. Horrible, que lo dejé, tomé dos sorbos, me salió cincuenta pesos y lo dejé ahí tirado, horrible, me fui a la sandwichería de al lado y me comí una milanesa completa. (Grupo 7)

El testimonio anterior manifiesta las demarcaciones simbólicas impuestas a los alimentos cuando su sabor y textura provocan repulsión acorde a las categorías culturales dominantes, y cómo también se erigen límites morales entre nosotros y ellos según la racionalidad atribuida a las elecciones

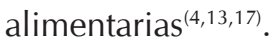

\section{CONCLUSIONES}

A lo largo de este artículo dimos cuenta de la complejidad que reviste contemporáneamente el acto alimentario. Los participantes de los grupos incorporan el conocimiento nutricional-científico en sus nociones de alimentación saludable. Sin embargo, también emergen cuestionamientos a algunas de las recomendaciones dominantes, especialmente entre aquellos que están más familiarizados con regímenes alimentarios alternativos y atentos a los debates entre los especialistas. Entre ellas, la conveniencia de consumir carne, aunque sea magra y su ingesta moderada, y productos light. También se plantean los riesgos para la salud de los alimentos procesados, más allá de la creciente atención pública a las grasas trans y al contenido de sal.

Si bien se valoran positivamente los alimentos orgánicos por su sabor, ausencia de toxicidad para el organismo y beneficios para el medio ambiente, se objetan sus costos y la localización del circuito comercial. Frente al nicho de elite que constituye actualmente la producción orgánica, en comparación con los alimentos convencionales producidos masivamente por la agroindustria, los participantes, con pocas excepciones, priorizan 
en sus decisiones de consumo los costos y la comodidad en el abastecimiento. Asimismo, mientras unos pocos promueven el consumo sustentable, otros, aunque comparten la preocupación por la calidad de los alimentos, relativizan el alcance de los esfuerzos individuales y familiares en la vigilancia alimentaria frente a otros riesgos a los que también están expuestos en la vida cotidiana. Entre ellos, la contaminación ambiental, cuyo control demanda políticas de responsabilidad corporativa y regulaciones estatales.

En una cultura alimentaria local muy carnívora se pone en duda la plausibilidad de dejar de comer carne, más allá de acordar en el rechazo hacia la crueldad con los animales, implicada en la industria agropecuaria. Los participantes priorizan el placer personal asociado al consumo de carne roja y el social, ligado a compartir determinados platos, especialmente en el ritual del asado. Las lógicas de consumo de los participantes ponen de manifiesto que alimentarse no solo es un acto nutricional y ético, sino también una práctica de disfrute y una ocasión social moldeada por la cultura gastronómica dominante. Se plantea, además, el esfuerzo que implica la reeducación alimentaria no carnívora para evitar incurrir en déficits nutricionales.
El conocimiento experto es un recurso que los participantes movilizan para protegerse de los mensajes publicitarios engañosos y las estrategias de mercadotecnia de la industria alimentaria. Les resulta necesario para interpretar el contenido de las etiquetas nutricionales, y para estar atentos a las controversias existentes en la comunidad científica sobre la alimentación apropiada. Sin embargo, los cambios en los consensos de la comunidad científica a lo largo del tiempo y el disenso en las recomendaciones de los especialistas redundan en más incertezas, e incluso en sospechas sobre los intereses comerciales con los que pueden estar alineados algunos consejos. En este contexto de incertidumbre, los participantes de los grupos demandan más información a través de campañas públicas masivas de promoción de la salud en materia alimentaria, y reconocen el rol que cumplen los medios de comunicación en la educación alimentaria. También esperan una mayor intervención del Estado para controlar los mensajes publicitarios de productos que se promocionan como protectores de la salud, cuya veracidad les genera dudas.

\section{AGRADECIMIENTOS}

El estudio que dio origen a este artículo fue financiado por la Secretaría de Ciencia y Técnica de la Universidad de Buenos Aires, a través del Proyecto UBACyT 20020110200178, “¿En búsqueda de la salud holística?: Discursos de cuidado de la salud, desigualdad social y estilos de vida". Matías Ballesteros, Mercedes Krause, Pablo Borda y Julia Bonetto integraron el equipo de investigación. Agradezco a Matías Ballesteros la lectura crítica del primer borrador de este artículo, y a los revisores anónimos por sus valiosos comentarios para mejorarlo.

\section{REFERENCIAS BIBLIOGRÁFICAS}

1. Menéndez EL. De sujetos, saberes y estructuras. Buenos Aires: Lugar Editorial; 2009.
2. Ward P, Henderson J, Coveney J, Meyer S. How do South Australian consumers negotiate and respond to information in the media about food and nutrition?: The importance of risk, trust and uncertainty. Journal of Sociology. 2007;48(1):23-41.

3. Organización Mundial de la Salud. Dieta, nutrición y prevención de enfermedades crónicas (Serie Informes Técnicos 196). Ginebra: Organización Mundial de la Salud; 2003.

4. Lupton D. Food, the body, and the self. London: SAGE; 1996.

5. Fischler C. Food, self and identity. Social Science Information. 1988;27:275-93.

6. Traversa O. Hábitos alimentarios y construcciones discursivas. En: Traversa O, (comp). Comer, beber, hablar: Semióticas culinarias. Buenos Aires: La Crujía; 2011. 
7. Lipovetsky G. La felicidad paradójica: ensayo sobre la sociedad de hiperconsumo. Barcelona: Anagrama; 2010.

8. Conrad P. Wellness as virtue: Morality and the pursuit of health. Culture, Medicine and Psychiatry. 1994;18(3):385-401.

9. Bourdieu P. La distinción: criterios y bases sociales del gusto. Madrid: Taurus; 1984.

10. Crawford R. Health as a meaningful social practice. Health. 2006;10(4):401-420.

11. Giddens A. Modernity and self-identity: self and society in the late modern age. Stanford: Stanford University Press; 1991.

12. Beck U. World risk society. Cambridge: Polity; 1991.

13. Lupton D. Food, risk and subjectivity. En: WiIlams SJ, Gabe J, Calnan M, (eds). Health, medicine and society. London: Routledge; 2000.

14. Fischler C. Las funciones de lo culinario. En: Piaggio LR, Solans AM, (comp). Enfoques socioculturales de la alimentación: Lecturas para el equipo de salud. Buenos Aires: Akadia; 2010.

15. Aguirre P. La construcción social del gusto del comensal moderno. En: Katz M, Aguirre P, Bruera M. Comer: Puentes entre la alimentación y la cultura. Buenos Aires: Libros del Zorzal; 2010.

16. Murcott A. Social influences on food choice and dietary change: a sociological attitude. Proceedings of the Nutrition Society. 1995;54(3):729-735.

17. Sassatelli R. Consumo, cultura y sociedad. Buenos Aires: Amorrortu; 2012.

18. Guptill A, Copleton D, Lucal B. Food and society: principles and paradoxes. Cambridge: Polity; 2012.

19. Lorenzen JA. Going green: The process of lifestyle change. Sociological Forum. 2012;27(1): 94-115.

20. Maye D, Kirwan J. Alternative food networks. Sociopedia.Isa. 2010. doi: 10.1177/205684601051.

21. Patel R. Obesos y famélicos: Globalización, hambre y negocios en el nuevo sistema alimentario mundial. En: Piaggio LR, Solans AM, (comp). Enfoques socioculturales de la alimentación: Lecturas para el equipo de salud. Buenos Aires: Akadia; 2014.

22. Patel R. Soberanía alimentaria: poder, género y el derecho a la alimentación. En: Piaggio LR,
Solans AM, (comp). Enfoques socioculturales de la alimentación: Lecturas para el equipo de salud. Buenos Aires: Akadia; 2014.

23. Johnston J, Szabo M, Rodney A. Good food, good people: Understanding the cultural repertoire of ethical eating. Journal of Consumer Culture. 2011;11(3):293-318.

24. Chen NN. Food, medicine, and the quest for good health: nutrition, medicine, and culture. New York: Columbia University Press; 2009.

25. Phelan JC, Link BG, Tehranifar P. Social conditions as fundamental causes of health inequalities: Theory, evidence, and policy implications. Journal of Health and Social Behavior. 2010;51(1):28-40.

26. Aguirre $P$. Estrategias de consumo: qué comen los argentinos que comen. Buenos Aires: Miño y Dávila; 2006.

27. Lupton D. Lay discourses and beliefs related to food risks: an Australian perspective. Sociology of Health and Illness. 2005;27(4):448-467.

28. Mintz S, Du Bois CM. The Anthropology of Food and Eating. Annual Review of Anthropology. 2002;31:99-119.

29. Krueger R. Focus group: A practical guide for applied research. London: Sage; 1988.

30. Liamputtong P. Focus group methodology: Principles and practice. London: Sage; 2011.

31. Sautu R. El Análisis de las clases sociales: teorías y métodos. Buenos Aires: Ediciones Luxemburgo; 2011.

32. Otamendi MA, Otero MP. Valoraciones sobre seguridad y tenencia de armas de fuego en Buenos Aires: un estudio con grupos focales. En: Sautu R, (comp). Práctica de la investigación cuantitativa y cualitativa: La articulación entre la teoría, los métodos y las técnicas. Buenos Aires: Lumiere; 2007.

33. Freidin B. Los límites de la solidaridad. Buenos Aires: Lumiére; 2000.

34. Maxwell JA. Qualitative research design: An interactive approach. Thousand Oaks: Sage Publications; 1996.

35. Bildtgard T. Trust in food in modern and late-modern societies. Social Science Information. 2008;47(1):99-128.

36. Waisbord. S. Perspectivas críticas en investigación y salud: ideas para investigaciones futuras. 
En: Petracci M, (coord). La salud en la trama comunicacional contemporánea. Buenos Aires: Prometeo; 2015.

37. Elliot C. Publicidad de alimentos para la diversión (fun foods): Descripción y análisis de los mensajes en los alimentos dirigidos a los niños/as en los supermercados. En: Piaggio LR, Solans AM, (comp). Enfoques socioculturales de la alimentación: Lecturas para el equipo de salud. Buenos Aires: Akadia; 2014.

38. Koch SL. A theory of grocery shopping: food, choice and conflict. London: Berg Publishers; 2012.

39. Aguirre P. La comida en los tiempos del ajuste. En: Torrado S, (dir). El costo social del ajuste (Argentina 1976-2002), Tomo II. Buenos Aires: Edhasa; 2010.

40. Arnould EJ, Thompson CJ. Consumer culture theory (CCT): Twenty years of research. Journal of Consumer Research. 2005;31(4):868-882.

41. Denham S. Relationships between family rituals, family routines, and health. Journal of Family Nursing. 2003;9(3):305-330.

42. Thompson C. Caring consumers: Gendered consumption meanings and the juggling lifestyle. Journal of Consumer Research. 1996;22:388-407.

43. Administración Nacional de Medicamentos, Alimentos y Tecnología Médica. Boletín para con- sumidores $\mathrm{N}^{\circ} 43$ [Internet]. 2010 [citado 10 ene 2016]. Disponible en: http://tinyurl.com/hl7gccz.

44. Wilson T. Understanding media users: from theory to practice. East Sussex: Wiley-Blackwell; 2009.

45. Contreras J. Patromonio y globalización: la identidad culinaria como respuesta. En: Piaggio LR, Solans AM, (comp). Enfoques socioculturales de la alimentación: Lecturas para el equipo de salud. Buenos Aires: Akadia; 2014.

46. Breihl J. La epidemiología crítica: una nueva forma de mirar la salud en el espacio urbano. Salud Colectiva. 2010;6(1):83-101.

47. Erbetta E. Del productor al vecino: crece la feria orgánica de Agronomía. La Nación. 3 ago 2015.

48. McKinlay JB. A case of refocusing upstream; The political economy of illness. En: Conrad P, (ed). The Sociology of Health and Illness: Critical Perspectives. New York: Worth Publishers; 2001.

49. Prüss-Üstün A, Corvalán C. Preventing disease through healthy environments: Towards an estimate of the environmental burden of disease. Geneva: WHO; 2006.

50. Archetti E. Hibridación, pertenencia y localidad en la construcción de una cocina nacional. Trabajo y Sociedad. 2000;2(II):1-13.

\section{FORMA DE CITAR}

Freidin B. Alimentación y riesgos para la salud: visiones sobre la alimentación saludable y prácticas alimentarias de mujeres y varones de clase media en el Área Metropolitana de Buenos Aires. Salud Colectiva. 2016;12(4):519-536. doi: $10.18294 /$ sc. 2016.913

Recibido: 30 de marzo de 2016 | Versión final: 28 de agosto de 2016 | Aprobado: 7 de octubre de 2016

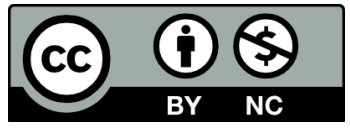

Este obra está bajo una licencia de Creative Commons Reconocimiento-NoComercial 4.0 Internacional. Reconocimiento - Permite copiar, distribuir y comunicar públicamente la obra. A cambio, se debe reconocer y citar al autor original. No Comercial - Esta obra no puede ser utilizada con finalidades comerciales, a menos que se obtenga el permiso. 